\title{
General Human Traits Oriented Generic Elastic Model for 3D Face Reconstruction
}

\author{
Joi San $\operatorname{Tan}^{1}$ \\ tjs11_com120@student.usm.my \\ Ibrahim Venkat $^{1}$ \\ ibra@usm.my \\ Iman Yi Liao ${ }^{2}$ \\ iman.liao@nottingham.edu.my \\ Philippe De Wilde ${ }^{3}$ \\ p.dewilde@kent.ac.uk
}

\author{
${ }^{1}$ Universiti Sains Malaysia, \\ Pulau Pinang, Malaysia. \\ ${ }^{2}$ The University of Nottingham, \\ Malaysia Campus, Semenyih, \\ Selangor, Malaysia. \\ 3 The University of Kent, \\ Canterbury Kent CT2 7NZ, \\ United Kingdom.
}

We propose a Simplified Generic Elastic Model $(S-G E M)$ which intends to construct a 3D face from a given $2 \mathrm{D}$ face image by making use of a set of general human traits viz., Gender, Ethnicity and Age (GEA). We hypothesise that the variations inherent on the depth information for individuals are significantly mitigated by narrowing down the target information via a selection of specific GEA traits. In this paper, we propose a $3 \mathrm{D}$ reconstruction method to retain the robustness of the PCA-based models and in the meantime to provide control over the depth values of $2 \mathrm{D}$ facial feature points. We formulate the reconstruction of the $3 \mathrm{D}$ face model of a given $2 \mathrm{D}$ face image as a posterior estimation of the PC coefficients $\Phi$ given the observations of the 2D facial feature points $x_{f}$. The depth value $Z$ of the $2 \mathrm{D}$ feature points is expressed as the hidden information. The posterior probability is represented as the marginal distribution of $P\left(\Phi \mid \boldsymbol{x}_{f}\right)$ integrated over $Z$ as shown below:

$P\left(\Phi \mid x_{f}, \Delta\right) \propto \sum_{Z_{f}} P\left(\Delta \mid x_{f}, Z_{f}\right) \cdot P\left(x_{f}, Z_{f} \mid \Phi\right) \cdot P(\Phi)$

where $\boldsymbol{x}_{f}$ represents the $x$ and $y$ coordinates of the $2 \mathrm{D}$ input feature points, $\Delta$ represents the corresponding GEA group and $Z_{f}$ represents the hidden $Z$ values of the 2D input feature points. Also, $\boldsymbol{x}_{f}$ and $\Delta$ are the observed variables in this representation. Based on the Bayesian theory, $P\left(\Delta \mid x_{f}, Z_{f}\right)$ can be written as,

$$
P\left(\Delta \mid x_{f}, Z_{f}\right)=\frac{P\left(x_{f}, Z_{f} \mid \Delta\right) P(\Delta)}{\sum P\left(x_{f}, Z_{f} \mid \Delta\right) P(\Delta)}
$$

$P\left(\boldsymbol{x}_{f}, Z_{f} \mid \Delta\right)$ is defined using simplified mixture model. The proposed S-GEM method was compared with the PCA-TR method [1] and a method of utilising the Z-coordinates of the

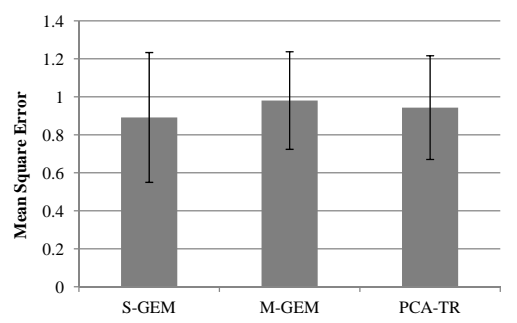

Figure 1: Empirical test of the proposed method (S-GEM), M-GEM and PCA-TR.

model mean-face feature points (we name it MGEM). M-GEM is chosen for comparisons as it is based on the same principle of the popular GEM model[2], i.e., using the model mean face depth value to represent any individual face depth. The results in Figure 1 shows that the proposed S-GEM method has produced the least MSE out of the three methods in terms of the full 3D face shapes. Figure 2 shows the outputs of the newly reconstructed 3D face models using proposed S-GEM.

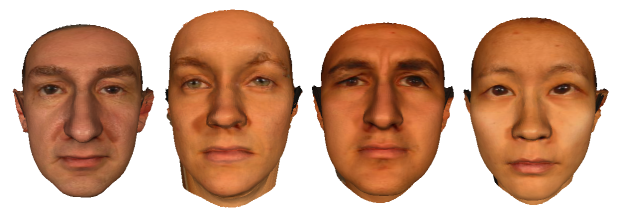

Figure 2: Outputs of the newly reconstructed 3D face models using proposed S-GEM.

[1] AY Maghari, Ibrahim Venkat, Iman Yi Liao, and Bahari Belaton. PCA-Based Reconstruction of 3D Face Shapes using Tikhonov Regularization. International Journal of Advances in Soft Computing and its Applications , 5(2):1-15, 2013.

[10] Jingu Heo. 3D Generic Elastic Models for 2D Pose Synthesis and Face Recognition, 2009. 